\title{
Modeling and Analysis of 3d Printing Ws-Bpel Business Processes Based on Servicenet
}

\author{
Cheng-Lei ZHANG ${ }^{1,2}$, Bu-Yun SHENG ${ }^{1 \#}$, Fei-Yu ZHAO ${ }^{1}$, Xi-Yan YIN ${ }^{1}$, Jing-Jing CAO ${ }^{1}$ \\ 1. School of Mechanical and Electronic Engineering, Wuhan University of Technology, Wuhan 430070, China; \\ 2. School of Mechanical \& Vehicle Engineering, Liyin University, Liyin 276005, China \\ E-mail: shengby@whut.edu.cn
}

\begin{abstract}
To solve the problem that whether the described Web service by business process execution language were interactive compatible, a method of WS-BPEL(Web Services Business Process Execution Language) parsing and execution was proposed. The service compatibility checking algorithm based on the Mediation model, which can provide multi-level service checking compatibility, and realize the goal of Service Cooperation or the demand of ValueAdded Services. Based on BPMN specification, a task modeling and management tool was proposed to support the service components for assembly component. It supports both Web service automatic retrieval and service content analysis based on QoS information, and the task execution model between the BPMN specification task descriptions was transformed into the BPEL specification task description model. Finally, a model transformation strategy based on meta-model mapping was put forward. The algorithm was designed and examples were given to demonstrate the efficiency of 3D Printing WSBPEL.
\end{abstract}

\section{Introduction}

In recent decades, many experts and scholars, as well as enterprises themselves from all over the world, have been devoted to exploring and studying how to solve the problem of a lack of capital, technology, personnel and equipment, so as to improve their business management model [1]. In order to improve the intangible assets and comprehensive competitiveness of enterprises, we propose an idea that transforms a vertical department management based on the principle of "division of labor" and "institutionalized management theory" into business process management based on cloud manufacturing (CMfg) in this paper [2]. For the changes in the external business environment and continuous improvement in the internal conditions, enterprises have led to a large number of new business processes [3].

Meanwhile, with the continuous development of key technologies of business management logic engine and $\mathrm{CMfg}$ service task management tool, CMfg service composition modeling arose at a timely moment. CMfg is defined by Li Bohu [4] as service-oriented, efficient, low consumption and knowledge-based networks for new manufacturing patterns and technology. Ye Yanming [5] came up with a social business process management and process recommendation method. The method was mainly used for mining process execution meta-paths that had high matching degree with the building process framework, which was conducive to the procedure convergence of social process modeling. Lu Jianfeng [6] put forward an integration framework based on Business Process Management (BPM). This framework was designed to provide unified modeling, executing and monitoring environment for various business processes within and cross enterprises. Business Process Execution Language for Web Services (BPEL4WS or BPEL) was used to define business service collaboration mode. Piero Fraternali [7] gave a model-Driven Approach to Social BPM Applications; this chapter has discussed the levels of adoptions of social features inside organizations and business process models, and examined the requirements that Social BPM poses to process specification languages.

This paper focuses on the Business Management Logic Engine. This engine is running in the cloud environment, which will do in response to the transaction with the increasingly wide range of manufacturing resources and manufacturing capacity. In order to deploy the manufacturing service business process file, the execution processing service business process processor realizes the interpretation and implementation of manufacturing service process will replace the concurrent processing in the eventbased concurrent processing mode to executes the expression framework and finally monitoring and scheduled business process. Taking the project requirements into consideration, a new business management logic engine of 
service resource network for intelligent manufacturing is applied to achieve the CMfg operation mode with multiple enterprises.

\section{The Business Management Logic Engine}

This paper focuses on the key technologies of business management logic engine and the architecture, such as dynamic service collaboration, task running supervision, transaction processing and so on.

\section{1 the key technologies of business management logic engine}

The specific research is as follows:

\section{1) Mediation-Based Dynamic service collaboration adaptation}

The main problem of service collaboration is how to ensure the consistency and correctness of the service cooperation, to realize the goal of service or the demand of value added services. The introduction of conversational services has brought new effects to the service coordination. For this, this paper firstly proposes that services participate in collaboration must satisfy the whole service collaborative requirement by using input/ output modules, and also need to meet the needs of the whole in the interactive protocol service level[8]. That is, the services invocation temporal relationships and the interaction between services and services must meet the requirements of accuracy and consistency (protocol compatibility).

According to the problems above, this paper proposes a top-down approach to achieve the service collaboration. This is as shown below:

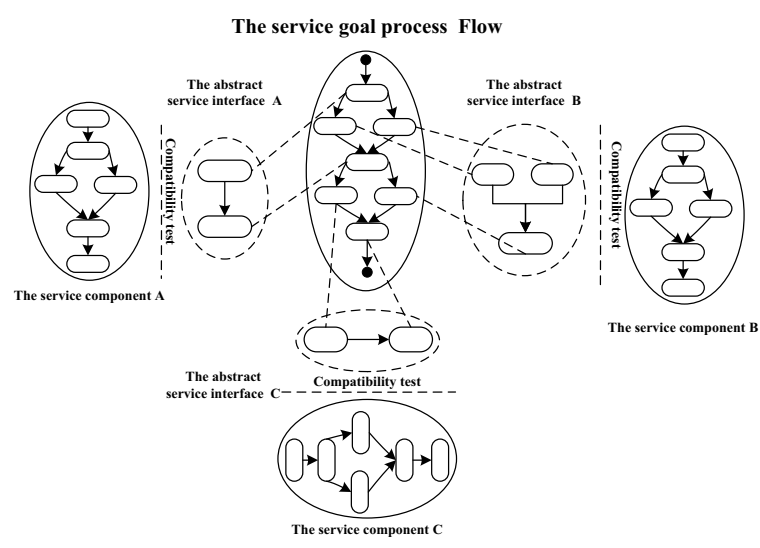

Figure 1. The Role-Based Distributed Compatibility Checking Method

Firstly, the global coordinated service process is strictly described as a contractual relationship of collaboration and public News between services. The formula of the message expression is shown as:

\author{
Choreography $=(\mathrm{M}, \mathrm{R}, \mathrm{PCHOR})$ \\ , and the contract relation is shown as: \\ STOP \\ $\mathrm{P}=\mathrm{a} \rightarrow \mathrm{P}|\mu \mathrm{X} \cdot \mathrm{F}(\mathrm{X})| \mathrm{P} \square \mathrm{Q}|\mathrm{P} \Pi \mathrm{Q}| \mathrm{P}$; Q | P\|Q | SKIP |
}

Finally, the characteristics of the interactive adapter are analyzed by Asynchronous communication method of abstraction task flow and service interface, so this paper will automatically generate an algorithm of automatic generation when fit under the premise of adaptive[9]. This system achieves to cooperation target through the distributed interaction of the adapter, and ensures to the correctness of cooperation by the interaction of adapters and service component. This method is as shown below:

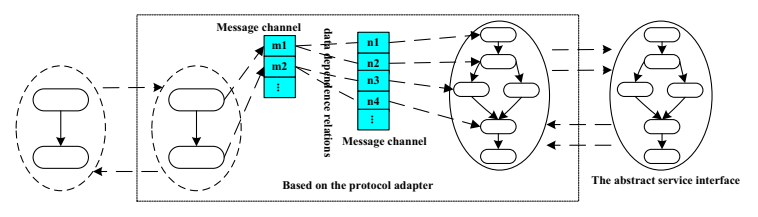

Figure 2. Adapter automatically generated model based on data dependence

The workflow of adapter automatically generated model based on data dependence is as shown:

First of all, this paper establishes a message channel that is supported data exchange under Bilateral agreement:

Pipe_mr $=[(\| \mathrm{i}<$ nleft?msi $) \rightarrow$ synth $\rightarrow$ right!mr $\rightarrow$ SKIP $]$ [close $\rightarrow$ SKIP]

Then, establishes an asynchronous communication protocol between POA and PSI according to the corresponding mapping relationship of message $M$ in the coordination model.

$$
(\| \mathrm{i}<\text { nPipe_mi }) / /[\text { POA_AC } \| \text { PSI_AC }]
$$

Finally, the adapter construction tool can be obtained by $(\| \mathrm{i}<$ nPipe_mi $) / /[$ POA $\cup$ AC $\|$ PSI $\cup \mathrm{AC}]$.

\section{2) Reliability Assurance Mechanism of service cooperation based on transaction}

Transaction is a key technology of distributed system reliability. The characteristics of Transaction are long time operation characteristics of service coordination, Crossdomain coordination, strict autonomy and heterogeneity of the Web service. The traditional transaction technology is difficult to be directly applied to reliability guarantee of service coordination. From the perspective of adaptative Services Cooperative transaction model, we study the key technology of relaxed atomic security, failure recovery and concurrency control of transactional services.

First of all, proposes a Transactional Service Coordination Model (TSCM) based on hierarchical. The model is described as the static composition of Transactional Service Coordination, including structure, attributes, and constraints and so on. The attributes of TSCM are divided into two groups: retrievable attributes and compensable attributes. According to retrievable and 
compensable attributes of TSCM, these attributes are divided the web services of different transaction behaviors into four classes, pivot, compensable, retrievable and trivial. And TSM are characterized by the status transfer diagrams.

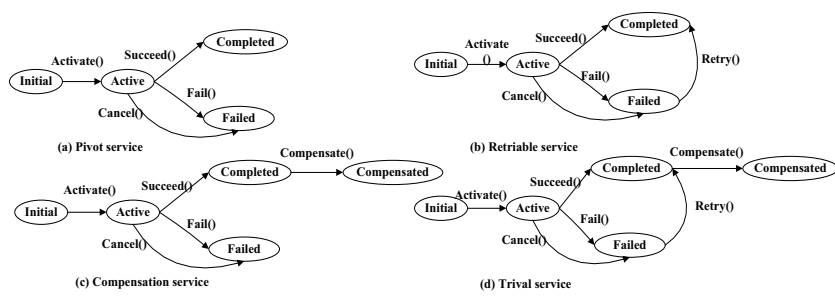

Figure 3. The state transition diagram of different types of services

Secondly, on the basis of TSCM model, we take advantage of the semantic information contained in the userdefined relaxed atomicity requirement and put forward a TSCM-FR. The TSCM-FR is a service cooperative failure recovery algorithm based on relaxation atomic driven. There is an exception handling mechanism based on TSCM-FR to capture the possible failures that allow the fault to be handled flexibly at different levels. This is as shown in the following figure [10]. When making atomic drive failure recovery, Multi-level exception handling system is based on the concept that the optimal configuration is a series of selection rules, and the service cooperation is finished by the automatic retrying and compensation.

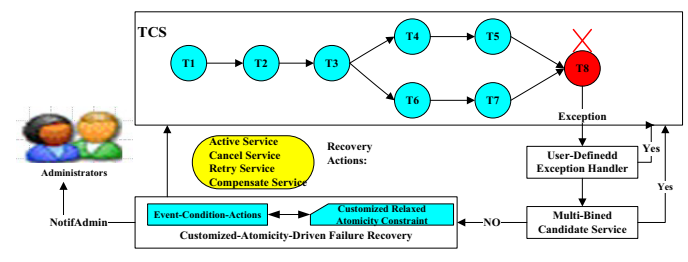

Figure 4. Multi-level exception handling system diagram

Thirdly, as a transactional service collaboration model, the back-end relaxes isolation among services, and the finished intermediate results are visible to the outside world. This relaxation makes the collaboration inconsistency for multiple transactional services in the execution of concurrent, and increases the difficulty of development. In order to solve this problem, we put forward a distributed concurrency control protocol based on transaction service collaboration model (TSCM-CC). In TSCM-CC, the service provider maintains a local dependency graph, which is synchronized with the service call. If the service call does not rely on any executed service, the direct execution is allowed; If the service call depends on the executed service, it needs to judge whether to establish a corresponding dependency relation, and then enter the execution again.

Finally, based on the above, we extend the syntax and deployment descriptor specification for the most popular service collaboration description language. This language is also called WS-BPEL [11-13]. This extension increases the transaction characteristics of the language, and the concept of Candidate Sphere is introduced to support of transaction and atomicity. On this basis, this paper realizes a transactional service collaborative support framework based on the AOP mechanism. This framework is as shown below
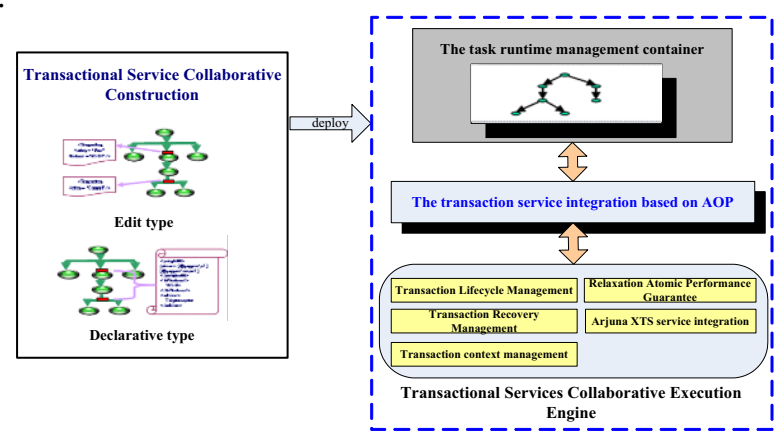

Figure 5. The transactional services collaborative support framework

\section{3) Situational-oriented monitoring and adaptive recovery mechanisms for service collaboration}

Because of the dynamic nature of service computing environment, it is possible to deviate from the execution of services collaboration and business objectives when the service collaboration is performed. The traditional design methods for analysis and verification cannot guarantee the correctness of the operating system. The service collaboration needs to be monitored of monitoring services at runtime, collects all kinds of situation information. Based on the pre-defined strategy, the dynamic adjustment of the collaborative structure is realized [13]. Based on this, the software system is correctly maintained and supported to the adaptive evolution of service coordination. Situation is a characterization of internal and external factors. The external factors include user needs and business environment changes. The internal factors include services coordination runtime under unexpected circumstances. The situation-oriented services collaboration includes the functional and nonfunctional aspects of service collaboration.

The definitions of Monitoring event definitions is:

$\mathrm{e}::=$ start $\mid$ end $\mid \mathrm{msg}$ (link.input/output = message)

The Collaborative Task Instance Monitoring Requirements Description Language is:

$\mathrm{b}::=\mathrm{e}|\mathrm{Yb}| \mathrm{Ob} \mid \mathrm{Hb}$ | b S b |! b | b Logop b|n Relop n | true $\mid$ false

$\mathrm{n}::=$ Count (b) $\mid$ Time (b) $\mid \mathrm{b}$ ? $\mathrm{n}: \mathrm{n} \mid \mathrm{n}$ Numop $\mathrm{n}|0| 1 \mid \ldots$

The Description Language of Collaborative Task Class Monitoring Requirements is:

B ::= And(b) | Y B | O B | H B | B S B | N Relop N | ! B | B Logop B | true | false

$\mathrm{N}::=\operatorname{Count}(\mathrm{b})|\operatorname{Sum}(\mathrm{n})| \operatorname{Avg}(\mathrm{n}) \mid \mathrm{N}$ Numop N $|0| 1 \cdots$

In view of services coordination, we put forward a kind of service collaborative monitoring method based on dynamic AOP technology, it is as shown in the figure below.

In the monitoring of functional aspects, the service collaborative monitoring method based on dynamic AOP technology is supported on the expression of complex 
events, and through the event tree to organize the hierarchical relationship between behavior constraint automaton and composite event automaton to correctly identify events, so as to effectively monitor the collaborative behavior, check whether the predefined constraint violation. In the monitoring of non-functional aspects, the service collaborative monitoring method based on dynamic AOP technology is collect all kinds of situation information, including the statistical service called time, service execution reliability, etc [14].

\section{2the architecture of business management logic engine}

The business management logic engine is an independent work engine that can be deployed on any Java/J2EE application server. The business management logic engine is supports in the form of WS-BPEL1.1, and its architecture is shown in figure:

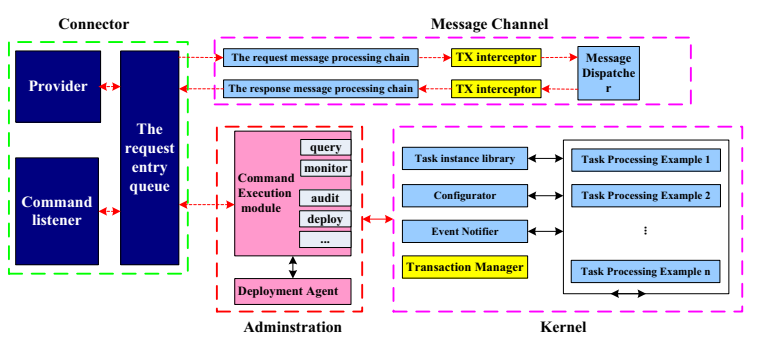

Figure 6. The Business logic engine management architecture diagram

The Business logic engine management mainly includes the following functional modules:

- Connector module: The message Provider module is responsible for receiving and sending SOAP messages.

- Message Channel module: The request message and response message processing chain module which support messages request and response before and after the customization of processing needs.

- Administration module: This module is the command execution module to achieve the deployment of the task, monitoring, query and other operations, and deployment agent module is responsible for parsing and deploy operating of tasks.

- Kernel module: The event notification module has an event notification mechanism based on Pub/Sub mode.

\section{New chaos optimal algorithm for BPMN}

Business Process Modeling and Notation, is called BPMN. It includes these entities are combined into a business flow chart. The Business Process Management Initiative has developed a set of standard that is also called BPMN. It defines a Business Process Diagram(BPD), which is based on a flowcharting that is designed to create a graphical model of business process operations. By the BPMN business process design specification, this paper has developed a platform task management tool based on model-driven[15]. It is a task modeling and management tool which supports the integrated component of service components. It provides a unified task modeling visualization development tool that allows users to use a graphical drag and drop so that easily, intuitively and effectively design, modification and maintenance of CMfg task flow, and WYSIWYG, which greatly improves the usability of the tool.

In this paper, we mainly focus on the key technologies of the composite choice of multi-service QoS constraints, service portfolio model conversion and others. It is as follows:

\subsection{The composite choice of multi- service QoS constraints}

The problem of composite choice of multiservice QoS constraints is real a difficult NP problem. The major difficulty is that the solution space is too large to completed in polynomial time. At present, many studies are optimizing the search algorithm, but the effect is not ideal. Although some studies have improve the speed of discovery, the accuracy of service discovery will not meet the needs of the users. In response to this problem, we firstly propose to a method of personalized modeling. It can track and learn the user's interests and behaviors, and can accept the user's various constraints.

Definition: The user's preference value of service $\mathrm{x}$ is the average of attributes of service ${ }^{w_{i}}$, the formula is as follows:

$$
\operatorname{Pref}(x)=\frac{1}{M(x)} \sum_{w_{i} \in x} \operatorname{Pref}\left(w_{i}\right)
$$

Definition: The user's preference value of service ${ }^{w_{i}}$, the formula is as follows:

$$
\operatorname{Pref}\left(w_{i}\right)=I\left(X\left(w_{i}\right) ; V\right)=\log \frac{P\left(X\left(w_{i}\right) \mid V\right)}{P\left(X\left(w_{i}\right)\right)}
$$

Where $w_{i}$ is the attributes of service $\mathrm{x}, \operatorname{Pref}(x)$ is the user's preference value of service $x$.

Based on the above-proposed formula, a personalized model is as follow:

$$
\operatorname{Pref}(x)=\frac{1}{M(x)} \sum_{w_{i} \in x} \log \frac{P\left(X\left(w_{i}\right) \mid V\right)}{P\left(X\left(w_{i}\right)\right)}
$$

On this basis, we propose a constraint model based on user personalization model and service association model. On the one hand, the constraint model is one of the most effective ways to reduce the search space and improve the service choices' efficiency, on the other hand the constraint model reflects the users' characteristics and 
preferences, it can find a more effective combination of service satisfaction.

Based on constrained model, we propose an efficient heuristic selection algorithm for composite services. Unlike the previous work, it isn't focus on the optimization of the discovery algorithm. On the user's point of view, it is efficiency to obtain service discovery when mining users' information.

\subsection{The transformation of service composition model}

Task Description Model(TDM) is geared to serving the needs of business people or domain exporters. In order to facilitate mutual understanding between business people, it is to describe the tasks that uses the standard graphic elements defined by the BPMN specification. Task Execution Model(TEM) is geared to serving the needs of technical staffs. There are great differences between the BPMN model and BPEL model in terms of structural features, expression forms and expression ability. Therefore, it is feasible to realize the automatic transformation from BPMN model to BPEL model [16]. A feasible way is to find a way of abstract expression that can shield the details of the model. Keep a model level transformation when abstract model is transformed. Then, based on semantic equivalence, the model transformation strategy is carried out mapping transformation on the basis of semantic equivalence. The model transformation diagram is as follows:

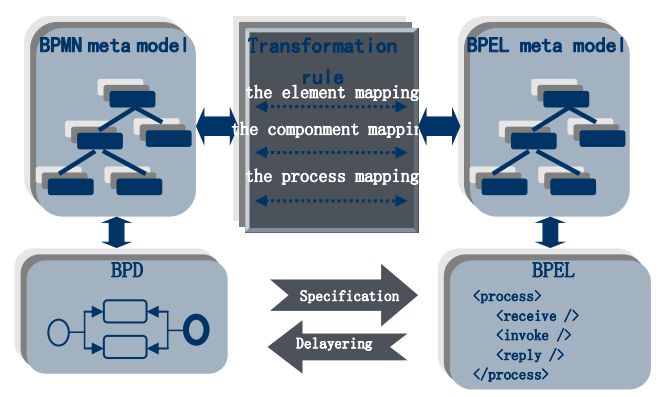

Figure 7. The model transformation diagram

In this paper, it is the mapping of metal model between the three levels of elements, components and processes. The mapping is as follows:

\section{3 the element mapping}

Element is the basic component elements of model. Since the semantics of BPMN is stronger than the semantic representation of BPEL, and therefore, in order to get the completed element matching in elements mapping ((i.e. for each element, an element can be found in the other model with the matching), we take the cutting strategy to get a set of core BPMN elements. Here, we have discussed this topic that the BPMN-based task model is the task model based on BPMN core elements [17].

\section{4 the component mapping}

According to the characteristics of the different componential structure, the paper makes several common mapping strategies. The component mapping is as follows:

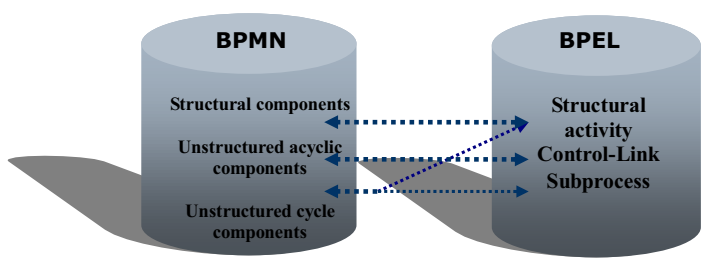

Figure 8. The component mapping diagram

For structural components, it can be directly mapped to the structure activities of semantic BPEL. As follows: Sequence、Switch、While、Pick、Flow.

\section{5 the process mapping}

The process mapping is on the basis of the component mapping and component specification. In short, component specification is a process by which components can be replaced by a simple task activity [18]. Through an iterative process of component specifications, process can be unceasing reduction, and ultimately the process mapping simplify it into a simple component . Finally, the whole process mapping is realized by component mapping.

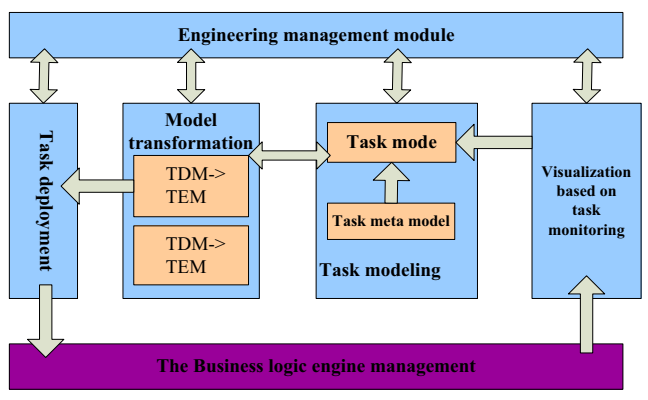

Figure 9. The architecture of platform task management tool

\section{Case study}

In order to verify the reliability and universality of the Business Process Management, and assess the performance and effect of this engine, we took a verification application in the $3 \mathrm{D}$ cloud printing creative and innovation product development. The service resources of the above-mentioned case may be released in our 3D Printing Colosseum manufacturing business process engine; moreover, the corresponding order/task workflows may be summarized in view of requirements of our case; and the following solution is given in view of the actual situations on how to carry out our research. The WS-BPEL snippet is as follow: 


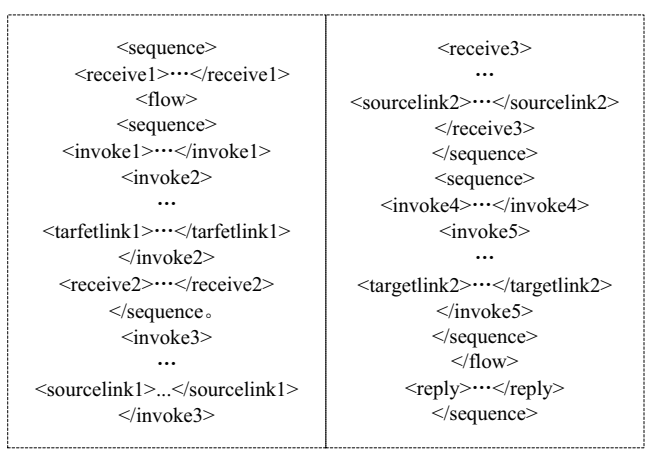

Figure 10. The WS-BPEL snippet

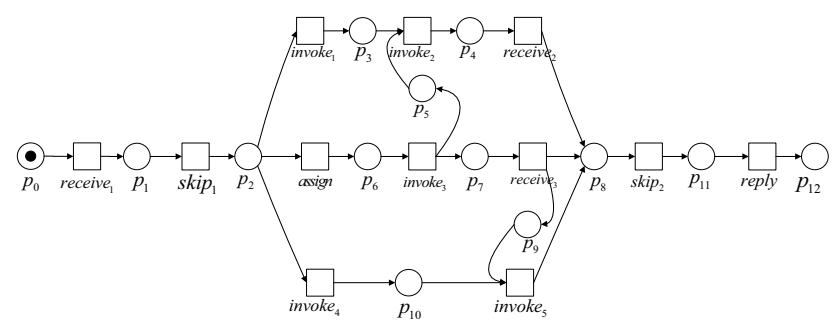

Figure 11. The logic representation

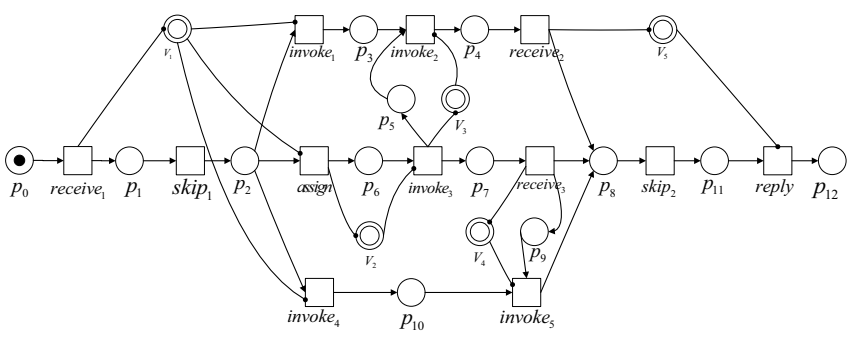

Figure 12. The semantic representation

In what follows, we have carried out several experiments to evaluate the impact of the heuristics for reducing the Web Services Business Process Execution Language and to prove the effectiveness of our approach in the 3D Printing Colosseum manufacturing business process[19]. We have also generated a WS-BPEL process composed by 12 activities, a set of 30 potential users, a role hierarchy of 6 roles, 3 separation of duty and 4 binding of duty and context constraints. Such 3D Printing Colosseum manufacturing business process has a MaxRoles value equal to 6 . We have considered four test cases that are summarized in Table 1. It is in the following cases:

a) we varied the number of potential user from 30 to 70 and kept the number of separation of duty and binding of duty and context constraints equal to 4 , and the value of MaxRoles is 6.

b) we varied the number of potential user to 30 and kept the number of separation of duty and binding of duty and context constraints equal to 4, and the value of MaxRoles is [3,5]. c) we varied the number of binding of duty constraints defined for the process from 0 to 5 and we set the number of separation of duty and context constraints to 4 , the value of MaxRoles to 6 and the number of potential users to 30 .

d) we varied the number of binding of duty and context constraints from 0 and set the number of separation of duty constraints defined for the process from 3 to 5, MaxRoles value to 6 and the number of potential users to 30 .

\section{TABLE I. TEST CASES PARAMETERS}

\begin{tabular}{cccccc}
\hline $\begin{array}{c}\text { Test } \\
\text { case }\end{array}$ & $\begin{array}{l}\text { Business } \\
\text { process }\end{array}$ & $\begin{array}{l}\text { Num } \\
\text { of } \\
\text { BoD }\end{array}$ & $\begin{array}{l}\text { Num of } \\
\text { SoD }\end{array}$ & MaxRoles & $\begin{array}{l}\text { Num of } \\
\text { Users }\end{array}$ \\
\hline 1 & $\begin{array}{c}12 \\
\text { activities }\end{array}$ & 4 & 4 & 6 & $30-70$ \\
2 & $\begin{array}{c}12 \\
\text { activities }\end{array}$ & 4 & 4 & {$[3,5]$} & 30 \\
3 & $\begin{array}{c}12 \\
\text { activities }\end{array}$ & {$[0,5]$} & 4 & 6 & 30 \\
4 & $\begin{array}{c}12 \\
\text { activities }\end{array}$ & 0 & {$[3,5]$} & 6 & 30 \\
\hline
\end{tabular}

\section{Conclusions}

In this paper, we first analyzed the applications of CMfg service composition modeling, concluded the key technologies of business management logic engine, new chaos optimal algorithm for BPMN, and proposed the model transformation diagram. Based on these works, a service compatibility checking algorithm based on adaptation mechanism with the service interface and source service component of Business Management Logic Engine was proposed to guide the multi-level service compatibility checking [21]. A Business Process Diagram (BPD) is also designed for graphical model of business process operations.

\section{Acknowledgments}

This work is partially supported by the National Key Technology Research and Development Program of China and Nature Science Foundation of Hubei Province, China (No. 2016YFB1101700) and (No. 2015CFA115).

\section{References}

[1] B. Q. Huang, C. H. Li, C. Yin, X. P. Zhao, Cloud Manufacturing Service Platform for Small and Medium-sized Enterprises, Int J Adv Manuf Techno, vol. 65 , No. 12, 2013, pp. 1261-1272. 
[2] T. X. Song, C. L. Zhang, B. Q. Huang, A cloud manufacturing service platform for small and medium enterprises, Computer Integrated Manufacturing Systems, vol. 5, No. 5, 2013, pp. 47-52,.

[3] Turetken, A. Elgammal, W. van den Heuvel, and M. Papazoglou, Capturing compliance requirements: A pattern-based approach. IEEE Software, special issue on Software Engineering for Compliance, vol. 29, No. 3, 2012, pp. 28-36.

[4] F. Tao, Y. J. Laili, L. Zhang, Z. H. Zhang, A.Y. C. Nee, QMAEA: a quantum multi-agent evolutionary algorithm for multi-objective combinatorial optimization, SIMULATION, vol. 90 , No. 2 , 2014 , pp. 182-204.

[5] Y. Ye, Y. Xu, J. Yin, et al, Social business process management and process recommendation method, Computer Integrated Manufacturing Systems, vol. 22 , No. 2 , 2016 , pp.413-421.

[6] X. W. Liu, Y. Yang, X. D. Xu, C. C. Li, et al, Research on profit mechanism of $3 \mathrm{~d}$ printing cloud platform based on customized products, Applied Mechanics \& Materials, vol. 703 , 2014 , pp. 318-322.

[7] C. M. Wei, C. L. Zhang, T. X. Song, et al, A Cloud Manufacturing Service Management Model and Its Implementation, International Conference on Service Science, ICSS), vol. 5 , No. 5 , 2013 , pp. 60-63.

[8] M. E. Rangiha, B. Karakostas, Process recommendation and role assignment in social business process management, Science and Information Conference. IEEE, 2014 , pp.810-818.

[9] S. Usuki, H. Kanaka, K. T. Miura, Generation and control of 3D standing wave illumination for wide-field high-resolution 3D microscopic measurement, International Journal of Precision Engineering and Manufacturing, vol. 14, No. 1,2013, pp.55-60.

[10] S. Moser, A. Martens, K. Gorlach, et al, Advanced Verification of Distributed WS-BPEL Business Processes Incorporating CSSA-based Data Flow Analysis, International Journal of Business Process Integration \& Management, vol. 4, No. 4,2007, pp.4759.

[11] T. S. Heinze, W. Amme, S. Moser, A Restructuring Method for WS-BPEL Business Processes Based on Extended Workflow Graphs, Business Process Management. Springer Berlin Heidelberg, vol. 2009, pp.211-228.

[12] Hildebrandt T. A Standard-driven Implementaion of WS-BPEL 2.0[J]. Grass \& Forage Science, 2017, 40(40):183-192.

[13] G. Hayashi, E. Chiappetta, Analysis of the Change in Farmer Service Requirement and Farmers' Collaboration Foundation Under the Agricultural Industrialization in Northwest Region-the Explanation of Traditional Cooperation Decline and Cooperation Difficult, Journal of Northwest A \& F University, vol. 71, No. 9, 2013, pp.100-101.
[14] C. L. Zhang, A Cloud Manufacturing Service Platform for Small and Medium Enterprises, MNaster dissertation, Dept of Mechanical Eng, Hubei University of Technology, Wuhan city, Mass, 2013.

[15] Q. Yin, C. H. Li, Modeling for personalized Web service selection with QOS constraints based on GA and fuzzy control, Computer Engineering \& Applications, vol. 43, No. 27,2007, pp.226-229.

[16] D. Margaris, C. Vassilakis, P. Georgiadis, Improving QoS delivered by WS-BPEL scenario adaptation through service execution parallelization, The, ACM Symposium. ACM, vol. 2016, 2016, pp.1590-1596.

[17] A. Barnawi, A. Awad, A. Elgammal, et al, An AntiPattern-based Runtime Business Process Compliance Monitoring Framework, International Journal of Advanced Computer Science \& Applications, vol. 7, No. 2, 2016, pp.1-22.

[18] R. Thullner, S. Rozsnyai, J. Schiefer, H. Obweger, M. Suntinger, Proactive business process compliance monitoring with event-based systems. In Enterprise Distributed Object Computing Conference Workshops (EDOCW), 15th IEEE International, vol. 2011, pp.429437.

[19] B. T. Nguyen, D. H. Nguyen, T. T. Nguyen, Translation from BPMN to BPEL, current techniques and limitations Symposium on Information and Communication Technology. ACM, vol. 2014, pp.2130.

[20] H. Dun, W. Zhao, Y. Huang, et al. Modeling and Analysis of WS-BPEL Business Processes Based on ServiceNet. 200815 th AsiaPacific Software Engineering Conference, vol. 2008, pp.265-272.

[21] Parimala N, Kohar R. A quality metric for BPEL process under evolution[C]// Eleventh International Conference on Digital Information Management. IEEE, 2017. 